\title{
Usefulness of anterior-half body cast using a soft brace or lumbar belt for spinal compression fracture: Toyohira orthopedic device with attachment
}

\author{
Katsuhiro Toda ${ }^{1 *}$, Takahiro Takayama ${ }^{2}$ and Kanzo Amano ${ }^{1}$ \\ ${ }^{1}$ Department of Orthopedic Surgery, Kitahiroshima Town Toyohira Hospital, Japan \\ ${ }^{2}$ Department of Surgery, Kitahiroshima Town Toyohira Hospital, Japan
}

\begin{abstract}
Purpose: We developed a new external fixation method for spinal compression fracture in which the patient wears only the anterior-half of a body cast and the cast is fixed by applying a soft brace, lumbar belt, or rib belt for rib fracture treatment on it.

Advantages: Toyohira Orthopedic Device with Attachment (TODA) can be prepared on the first examination day. TODA does not cause a feeling of pressure and sleep disorder. TODA can be regarded as a simple Jewett brace capable of continuous attachment. By loosening TODA attachment by loosening the soft brace, lumbar belt, or rib belt before sleep, some patients are able to wear TODA by themselves when they stand up. Physicians are not called at night-time or on holidays for a feeling of pressure and sleep disorder. Using TODA, continuous cast attachment is easy even for elderly patients. TODA is also applicable for outpatients. Either treatment with a rigid brace, soft brace, lumbar belt after completion of treatment with TODA, or continuation of TODA can be selected. The number of casts can be reduced when the posterior-half of the cast is absent or thin. An anterior-half body cast can be made in the supine position. A soft brace is often prepared for elderly patients with spinal compression fracture on the first examination at another hospital. TODA can be prepared for such patients. Unnecessary body casts with various sizes after completion of treatment can be reused for other patients.
\end{abstract}

Supplement: When a soft brace is used, its widening by adding new string is necessary and a free sample or used one left in hospital is used, or those which became unnecessary after treatment completion are collected from several patients and stored. For a lumbar or rib belt, the belts after completion of TODA are reused for other patients.

Conservative treatment of spinal compression fracture includes external fixation with a soft brace, rigid brace, and body cast [1]. A conventional circumferential body cast is advantageous in that it is applicable on the first examination day and has a strong fixing force. However, it gives a feeling of pressure to the patient and disturbs sleep, and patients may request remodeling of the body cast, such as partial cut, or its removal for night-time and holidays, being disadvantages. A soft brace, which requires a casting, causes these problems rarely, but the completion from the first examination takes time and the fixing force is weak. A lumbar belt does not require a casting and can be attached from the first examination day without a feeling of pressure or sleep disorder, but the fixing force is almost none. There are various types of rigid brace requiring a casting, and several types may give a feeling of pressure and disturb sleep. The most disadvantageous point of a rigid brace is that the completion from the first examination takes time. When external fixation is necessary to treat a spinal compression fracture, it is necessary immediately after injury. External fixation methods to fix spinal compression fractures have advantages and disadvantages.

To supplement these disadvantages, we developed a new external fixation method in which the patient wears only the anterior-half body cast and the cast is fixed by applying a soft brace, lumbar belt, or rib belt for rib fracture treatment on it (Figure 1). The preparation procedure is as follows: 1) The patient wears a cotton tubular padding for a cast (stockinette) on the trunk and then an undercast padding.
2) The trunk is then covered with a body cast. The posterior-half may be thin or absent because it will be discarded. 3) One side of the cast is cut and the body cast is removed, followed by cutting the other side. This process is unnecessary when the posterior cast is absent. 4) The abdominal region of the cast is hollowed out (Figure 2), the upper and lower hitting regions are cut, and the right angled 4 corners are made rounded. 5) The undercast padding used in 1) is fixed with a tape to the posterior surface of the cast. 6) The body cast is covered with the cotton tubular padding for a cast (stockinette) used in 1) (Figure 2). 7) The body cast is then fixed with a soft brace, lumbar belt, or rib belt (Figure 1). When a soft brace is used, its widening by adding new string is necessary (Figure 3).

Toyohira Orthopedic Device with Attachment (TODA) solved various problems: 1) TODA can be prepared on the first examination day. 2) Conventional body casts cause a feeling of pressure and sleep disorder, but TODA normally does not cause these. Wounds may be

${ }^{*}$ Correspondence to: Katsuhiro Toda, Department of Orthopedic Surgery, Kitahiroshima Town, Toyohira Hospital, 4705, Azaka, Kita-Hiroshima Town, Yamagata-Gun, Hiroshima, 731-1222, Japan; Tel: +81-826-84-1155; Fax: +81826-84-1015; E-mail: goutattack@yahoo.co.jp

Key words: Spinal compression fracture, anterior-half body cast, soft brace, lumbar belt, rigid brace

Received: April 20, 2018; Accepted: May 14, 2018; Published: May 17, 2018 


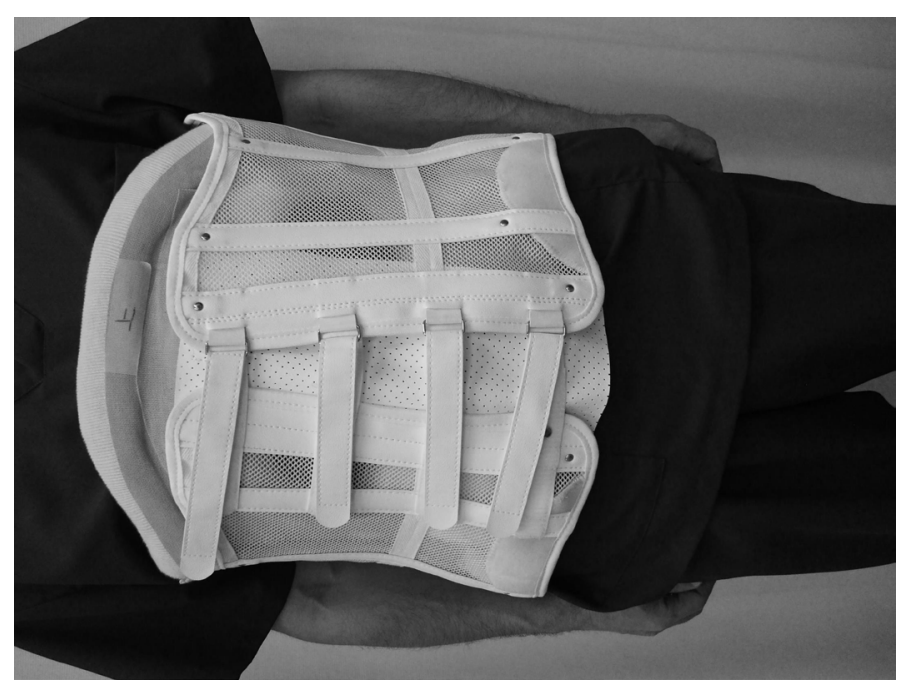

Figure 1. The first author wearing an anterior-half body cast fixed with a soft brace

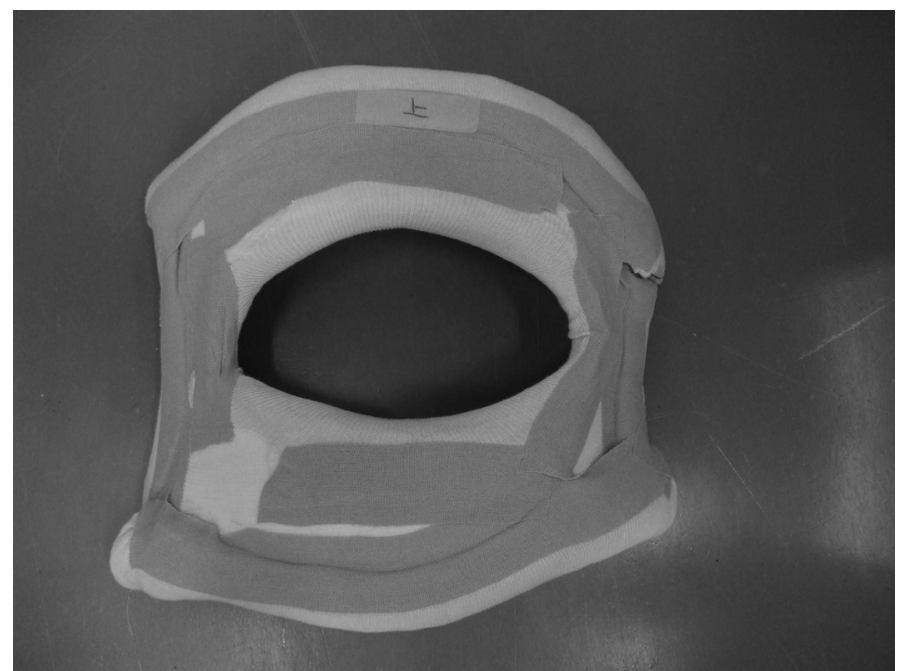

Figure 2. A used undercast padding is fixed with tape to the posterior surface of the cast The body cast was covered with a used cotton tubular padding for a cast (stockinette). The character indicated by an arrow means 'upside'

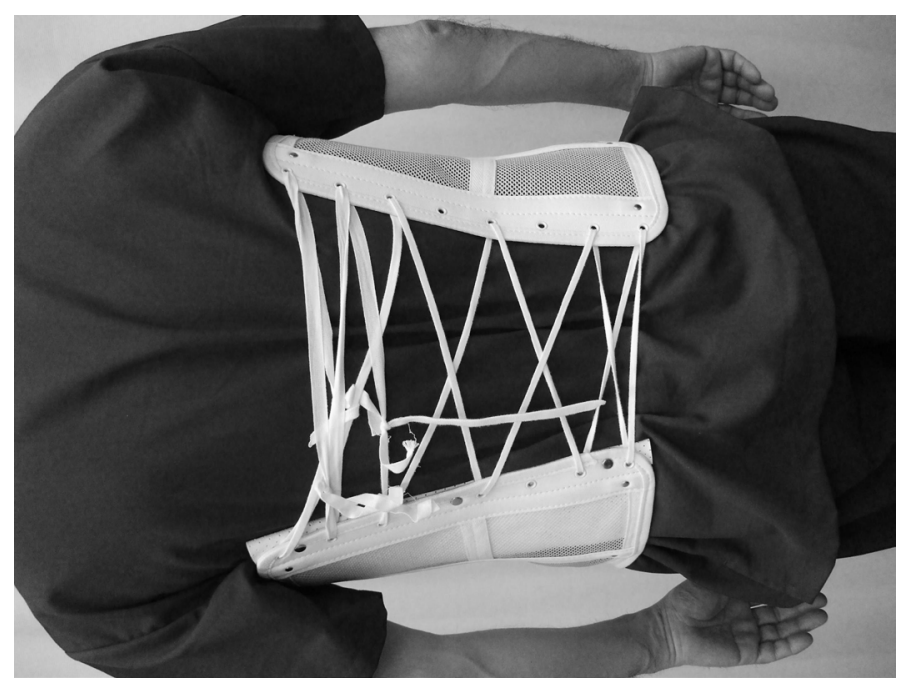

Figure 3. The soft brace was widened by adding new string. made by the cast edges and pressure ulcer may occur in rare cases, but these do not occur with TODA. Generally, the quality of life of patients was improved. 3) Jewett braces are not applicable to a small number of patients because of a feeling of pressure. It is appropriate to consider that TODA cannot be applied to such patients. Therefore, TODA serves as a touchstone to identify applicability of Jewett brace, which prevents preparation of an unusable Jewett brace. When Jewett braces are not applicable, a soft brace is prepared. 4) TODA can be prepared by anybody who has techniques to prepare conventional body casts. Preparation of TODA is easier than that of conventional body casts because the body cast is processed away from the patient's body. 5) When a casting of orthosis on a later day is necessary or pruritus is present, TODA can be immediately removed. 6) TODA can be regarded as a simple Jewett brace capable of continuous attachment. 7) By loosening TODA attachment by loosening the soft brace, lumbar belt, or rib belt before sleep, some patients are able to wear TODA by themselves when they stand up. 8) Physicians are not called at night-time or on holidays for a feeling of pressure and sleep disorder, showing that TODA is beneficial for both patients and physicians. 9) It is often difficult for elderly patients to continuously wear a conventional body cast, and such cases have been treated with a soft brace alone. Using TODA, continuous cast attachment is easy even for elderly patients. TODA is also applicable for outpatients. 10) It may be difficult to judge whether spinal compression fracture is fresh in institutions having no MRI. The use of TODA for patients later clarified as having no fracture is not problematic. 11) Either treatment with a rigid brace, soft brace, lumbar belt after completion of treatment with TODA, or continuation of TODA can be selected. 12) The number of casts can be reduced when the posteriorhalf of the cast is absent or thin. 13) An anterior-half body cast can be made in the supine position. It is valuable for patients who cannot stand due to severe pain. 14) As described below, Japanese national health insurance does not cover rigid brace preparation in patients for whom a soft brace has been prepared. A soft brace is often prepared for elderly patients with spinal compression fracture on the first examination at another hospital. TODA can be prepared for such patients. 15) Used unnecessary body casts with various sizes after completion of treatment may be reused for other patients. We started TODA more than one year ago. Many spinal compression fracture patients who used TODA discharged. As a results, we have many anterior-half body casts with various sizes and shapes. When non-orthopedic surgeons have to treat patients with spinal compression fracture at night or on holidays, TODA can be applied using the used unnecessary body cast. At a later date, anterior-half body cast is made to fit on the body of each patient. 16) When a patient with spinal compression fracture visits our hospital after 4 p.m. on weekdays, TODA is applied using the used unnecessary body cast. At a later date, anterior-half body cast is made to fit on the body of each patient. It reduced overtime of staff including physicians. 17) TODA can be applied to patients who underwent spinal surgery.

The biggest problem with TODA is the body cast-fixing method, i.e., a soft brace is not always available. When a soft brace is used, a free sample or used one left in hospital is used, or those which became unnecessary after treatment completion are collected from several patients and stored. For a lumbar belt or rib belt requiring no casting, the belts after completion of TODA are reused for other patients. If a lumbar belt is used after completion of TODA with a lumbar belt, a new one with an appropriate size, not the one used, is used. In Japan, the combination of a body cast and soft brace preparations or a body cast and rigid brace preparations for the same patient is covered by health insurance, but the combination of rigid and soft brace preparations is not covered. When a soft brace cannot be used for TODA, it can be 
substituted by a lumbar belt. Bandage fixation is simple and applicable any time, but it readily becomes loose and needs much help from a nurse during application.

The force of Jewett braces to limit lumbar vertebral extension is weak. TODA is not applied when a circumferential cast or orthosis is necessary. We consider that a circumferential body cast or rigid brace is unnecessary for conservative treatment of spinal compression fracture and the Jewett brace is sufficient. There may be an opinion that a circumferential body cast or rigid brace is necessary for spinal compression fracture. If so, a circumferential body cast should be attached from the first examination day even in elderly patients and a circumferential rigid brace should be subsequently attached.

\section{Limitations}

The usefulness or unnecessity of external fixation (body cast, rigid brace, and soft brace) as conservative treatment of spinal compression fracture is not discussed. This report was written on the premise that external fixation is useful for conservative treatment of spinal compression fracture. It is obvious that TODA is better than a conventional circumferential body cast in terms of comfort. Therefore, from an ethical point of view, it is impossible to compare TODA and a conventional body cast. As a result, it is impossible to take data to show comfort.

\section{Conclusion}

TODA is a low-cost method applicable to many patients by a simple procedure, and it improves the quality of life of patients and reduces physician's labor.

\section{Acknowledgement}

We thank Medical English Service for reviewing this manuscript.

\section{Reference}

1. Stadhouder A, Buskens E, Vergroesen D, Fidler MW, de Nies F, Oner FC (2009) Nonoperative treatment of thoracic and lumbar spine fractures: a prospective randomized study of different treatment options. J Orthop Trauma 23: 588-594. [Crossref]

Copyright: (C2018 Toda K. This is an open-access article distributed under the terms of the Creative Commons Attribution License, which permits unrestricted use, distribution, and reproduction in any medium, provided the original author and source are credited. 